\title{
Fluid dynamics of COVID-19 spread
}

\author{
Azar Eslam-Panah $\odot$ \\ Penn State University at Berks, Reading, Pennsylvania 16910, USA
}

(Received 5 August 2021; published 15 November 2021)

\begin{abstract}
This paper is associated with a poster winner of a 2020 American Physical Society's Division of Fluid Dynamics (DFD) Milton van Dyke Award for work presented at the DFD Gallery of Fluid Motion. The original poster is available online at the Gallery of Fluid Motion, https://doi.org/10.1103/APS.DFD.2020.GFM.P0017.
\end{abstract}

DOI: 10.1103/PhysRevFluids.6.110502

The spread of the airborne COVID-19 virus has been causing significant loss of lives as well as financial losses in various industries throughout the world. Face masking and social distancing are widely recommended to minimize the spread of the virus from coughs, sneezes, regular breathing, and talking. Therefore, understanding the physics of the effectiveness of face masks and social distancing against airborne viruses is more important than ever [1]. Outward flow in breathing, coughing, and sneezing releases a turbulent cloud of hot and moist air containing suspended virus droplets trapped within it. This cloud is self-similar along its trajectory away from the mouth and can travel up to $8 \mathrm{~m}$ in the case of sneezes [2]. Suspended droplets which are denser than the air circulate within the cloud until they settle out and reach a final position. Droplets are able to stay in the air for minutes to hours and spread throughout a room and, possibly, entering ventilation systems. In reality, these droplets may land on healthy individuals and result in direct transmission or land on any surfaces which can lead to infection when a healthy individual touches those surfaces.

A face mask provides self-protection by filtering virus particles that would otherwise be inhaled by an uninfected person. They can also provide protection for others by trapping virus droplets expelled by an infected person [3]. The effectiveness of a face mask during inhalation and exhalation depends on the filtering material, the fit of the mask on the face, and the related leaks from the perimeter of the face mask. These effects were studied by placing various masks on Styrofoam mannequin heads mounted in a water tank and injecting dye through the mouth to demonstrate how differently facial masks perform and outline the paths of droplets. Figure 1 provides a lateral view of two mannequin heads with fluorescent dye injected from the mouth into the water in the presence of green laser light. Through dye flow visualization, it was revealed that without a face mask, the exhaled cloud moves in a horizontal direction and extends its influence through the whole region. It is shown that a cloth-made face mask can avoid direct spread of droplets, but the outflow redirects towards the edges of the mask where it inclines downward and leaks through the top and bottom edges of the mask [see Fig. 1(b)]. Thus, a better fit mask with better filtration materials does not let the outflow bypass the mask, and the speed of the outflow is reduced, therefore, it limits the region affected by exhalations to the nearby people.

During inhalation, a low-pressure region is developed between the mask and the face, thereby sealing, or reducing perimeter leaks. Hence, self-protection depends primarily on the face-mask

Published by the American Physical Society under the terms of the Creative Commons Attribution 4.0 International license. Further distribution of this work must maintain attribution to the author(s) and the published article's title, journal citation, and DOI. 


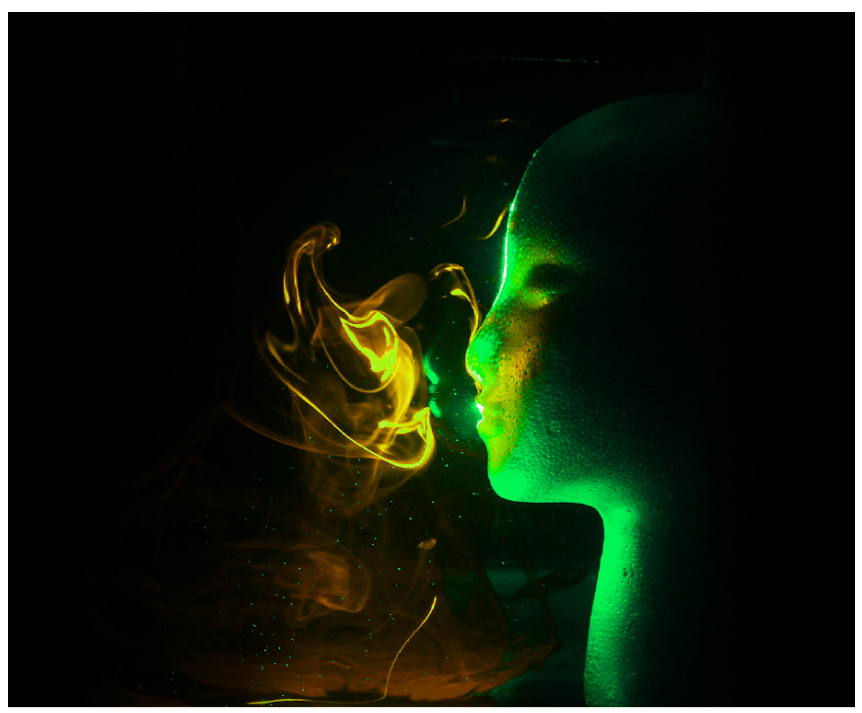

(a)

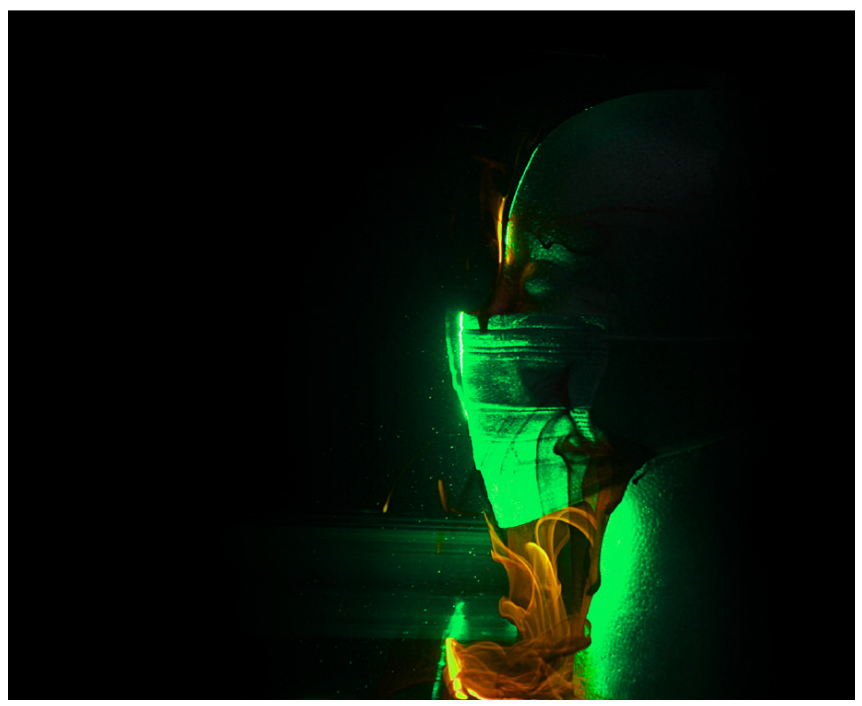

(b)

FIG. 1. Simulated spread of exhalation particles after the cough under indoor conditions (a) without a face mask and (b) with a cloth-based face mask. For the poster associated with this image see: https://doi.org/ 10.1103/APS.DFD.2020.GFM.P0017.

filtering material, and an increase in fiber density of the mask enhances the filtration efficiency [4]. During exhalation, the increased pressure in the region between the mask and the face pushes the face mask outwards, resulting in increased perimeter leakage. Although a mask can significantly reduce the velocity of the outflow jet, the leakage jets that issue from the perimeter can be turbulent and highly directed, potentially dispersing particles in vertical directions. During coughing and sneezing which generates high transient velocities, the effectiveness of face masks for others depends on the filtration materials as well as the fit of the mask. It has been shown that during 
indoor coughing some particles could travel up to $0.48 \mathrm{~m}$ with medical grade masks, $0.73 \mathrm{~m}$ with nonmedical grade masks, and $2.62 \mathrm{~m}$ without facial masks [5].

In an indoor environment, results suggest that wearing a well-fitted N95 mask can stop the tiny droplets from spreading during coughing or sneezing but not the cloth-based masks; however, the cloth-based masks are able to limit the speed of the outcoming jets significantly [6]. Hence, a reasonably well-fitted face mask with good filtration materials is necessary to prevent the spread of COVID-19 in indoor environments. However, this requires a greater inhalation effort by the person who wears it, and an appropriate balance between a proper structural design and the filtering material must be achieved. Under outdoor conditions with a mild breeze, leakage flow through the mask can cause particles to be entrained into the energetic shear layers around the body and transported at long distances by the turbulent flow. Although the particles are convected further, they are also greatly diluted by the turbulence present as it mixes with fresh air, hence, masking is generally not viewed as necessary in outdoor environments, unless in a crowded location where one is mixing with already potentially contaminated air.

Social distancing can provide a significant decrease in the risk of infection. In the absence of any face protection, the risk of infection halves at a 1-m distance and is still significant at a 2-m distance. It is obvious that the probability of contagion decreases with distance, and a strong risk reduction is obtained at a distance of several meters [6]. Wearing a suitable facial mask strongly decreases the risk of infection even at much smaller distances. In other words, social distancing according to the World Health Organization guidelines, remains necessary, although not sufficient, to measure to reduce the virus spread.

This paper was mostly qualitative and tried to visualize the rationale behind social distancing and face-masking guidelines. Future experiments should provide quantitative analysis of the leakage of the outflow jets, the particle dispersion through the jet, as well as the fluid-structure interaction of the mask during a variety of respiratory events. Ultimately, experimental and computational analyses should drive design changes that increase mask performance and provide data that inform guidelines on practices, such as social distancing.

I would like to thank C. Kovar and L. Panczner for helping with the images. I would also like to acknowledge support by the National Science Foundation with Award No. CBET-1903312 for supporting the PHOTO $321 \mathrm{~N}$ flow visualization course at Penn State University at Berks.

[1] L. Bourouiba, The fluid dynamics of disease transmission, Annu. Rev. Fluid Mech. 53, 473 (2021).

[2] N. H. L. Leung, D. K. W. Chu, and E. Y. C. Shiu, Respiratory virus shedding in exhaled breath and efficacy of face masks, Nat. Med. 26, 676 (2020).

[3] R. Mittal, R. Ni, and J. H. Seo, The flow physics of COVID-19, J. Fluid Mech. 894, F2 (2020).

[4] A. C. K. Lai, C. K. M. Poon, and A. C. T. Cheung, Effectiveness of facemasks to reduce exposure hazards for airborne infections among general populations, J. R. Soc., Interface 9, 938 (2012).

[5] A. Khosronejad, C. Santoni, K. Flora, Z. Zhang, S. Kang, S. Payabvash, and F. Sotiropoulos, Fluid dynamics simulations show that facial masks can suppress the spread of COVID-19 in indoor environments featured, AIP Adv. 10, 125109 (2020).

[6] G. Seminara, B. Carli, G. Forni, S. Fuzzi, A. Mazzino, and A. Rinaldo, Biological fluid dynamics of airborne COVID-19 infection, Rend. Lincei Sci. Fis. Nat. 31, 505 (2020). 